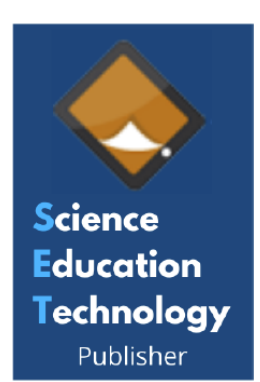

\title{
The Implications of Fruit and Vegetable Consumption in Patients with Diabetes
}

\author{
Anne Misher ${ }^{1}$, Chelsea A. Keedy ${ }^{2}$, Mackenzi Lee Meier ${ }^{3,}{ }^{*}$ and Phillip Greenspan ${ }^{1}$ \\ ${ }^{1}$ University of Georgia College of Pharmacy, Athens, GA 30602, Georgia \\ ${ }^{2}$ Clinical Pharmacy Specialist Ambulatory Care, St. Joseph's/Candler Health System, Savannah, 31419, Georgia \\ ${ }^{3}$ PGY2 Pharmacy Residency Ambulatory Care, St. Joseph's/Candler Health System, Savannah, 31419, Georgia
}

\section{Article Info:}

Keywords:

diabetes,

flavonoids,

pharmacology,

nutrition

Timeline:

Received: March 03, 2021

Accepted: May 11, 2021

Published: June 02, 2021

Citation: Misher A, Keedy CA, Meier ML,

Greenspan P. The Implications of Fruit and Vegetable Consumption in Patients with Diabetes. J Pharm Nutr Sci 2021;

11(1): 28-32.

DOI: https://doi.org/10.29169/1927-5951.2021.11.04

\begin{abstract}
:
It is widely accepted that the management of diabetes should include both pharmacologic and lifestyle modifications. However, these recommendations are not readily or consistently incorporated into clinical practice. Current guideline recommendations encourage an emphasis on nutrient-dense foods, which include those foods that tend to be high in flavonoids such as fruits and vegetables. Polyphenolic compounds in fruits and vegetables have been shown to affect the same biological processes as certain classes of pharmacological therapy used in the treatment of diabetes. A better understanding of the benefits of these compounds may help healthcare professionals, including pharmacists, communicate dietary recommendations to patients.
\end{abstract}

${ }^{*}$ Corresponding Author

E-mail: meierm@sjchs.org

(c) 2021 Misher et al.; Licensee SET Publisher.

This is an open access article licensed under the terms of the Creative Commons Attribution Non-Commercial License (http://creativecommons.org/licenses/by-nc/3.0/) which permits unrestricted, non-commercial use, distribution and reproduction in any medium, provided the work is properly cited. 


\section{INTRODUCTION}

The ideal management of diabetes is known to include both pharmacologic and lifestyle modifications [1,2]. It is optimal for patients to receive Medical Nutrition Therapy from a registered dietician. Medical Nutrition Therapy allows for a tailored and patient specific diet plan or suggestions to help with the symptoms and comorbidities of certain disease states [1]. Additionally, the entire health care team should be involved in the implementation of therapy to help patients achieve their goals. Current recommendations from the American Diabetes Association encourage an emphasis on nutrient-dense foods [1]. Nutrient dense foods include those foods which tend to be high in flavonoids such as fruits and vegetables. The American Association of Clinical Endocrinologists and American College of Endocrinology also recommend that patients with diabetes consume a plant-based diet that is high in fiber and phytochemicals/antioxidants while low in calories and glycemic index [2]. Better understanding of the benefits of these compounds may help healthcare professionals communicate dietary recommendations to patients.

\section{METHODS AND MATERIALS}

Two important clinical studies revealed that fruit consumption has significant beneficial effects on the progression of diabetes related complications. In 2014, Mahoney and Loprinzi studied the relationship between the consumption of whole fruits and vegetables in patients with diabetes and the extent of diabetes related retinopathy. After determining the patient intake of flavonoids, polyphenolic antioxidant compounds found in fruits and vegetables, they utilized the ETDRS (Early Treatment Diabetic Retinopathy Study) grading criteria to assess retinopathy [3]. The consumption of flavonoids was correlated with a significant decrease in diabetes biomarkers (C-reactive protein, hemoglobin A1C and fasting glucose) and reduced odds of diabetes related retinopathy by $30 \%$ [3]. Recently Du et al. (2017) performed a 7-year prospective study on approximately 500,000 Chinese adults [4]. Approximately 30,000 individuals were diagnosed at the start of the study with diabetes. They found that people with diabetes who consumed a high whole fruit diet had lower risks of microvascular (0.72 [0.61-0.87]) and macrovascular complications (0.87 [0.82-0.93]) and reduced all-cause mortality (adjusted $\mathrm{HR}=0.83$ [95\% Cl 0.74-0.93] $(p<0.001)$ [4].

Current pharmacological therapy may decrease mortality or the complications associated with diabetes by lowering plasma glucose concentrations and hemoglobin A1C [5,6]. Interestingly, certain polyphenolic compounds found in fruits and vegetables also affect the same biological processes. Numerous phytochemicals and extracts of a rather large variety of plants inhibit alpha-glucosidase and alpha-amylase limiting peak postprandial glucose absorption from the gastrointestinal tract, an effect similar to that of acarbose [7,8]. Phlorizin, a major polyphenolic compound present in apples, has been shown to inhibit sodium-glucose cotransporter-2 (SGLT2), reducing renal glucose reabsorption and promoting excretion of glucose mimicking the activity of the SGLT2 inhibitors (SGLT2i) [9,10]. Increased expression of PPARgamma, similar to that observed with thiazolidinediones, has been observed with green tea (epigallocatechin gallate) while inhibition of dipeptidyl peptidase 4 (DPP-4) mediated by the DPP-4 inhibitors (DPP-4i) can also be observed by several plant species $[11,12]$. These observations suggest that phytochemicals from fruits and vegetables may moderate the disease state in a manner similar to that observed with pharmacological therapy. While incorporating these food groups into one's diet alone would not lead to the efficacy seen with appropriate pharmacotherapy, the similar mechanism shared by these compounds lends credibility to their use in successfully augmenting the therapeutic benefits seen with pharmacotherapy.

\section{RESULTS AND DISCUSSION}

The mechanism by which plasma glucose lowering agents decrease the risk of diabetes related complications is due to the fact that there is less substrate for the non-enzymatic glycation and glycooxidation of proteins that occurs in nature, whether it is in occurring in food or in the human body [13]. This modification of proteins, classically studied by Maillard and Amadori, is now referred to as advanced glycation endproducts (AGE) [13-15]. This degree of glycation in patients with diabetes can be determined by the measurement of plasma hemoglobin $\mathrm{A} 1 \mathrm{C}$ or measuring skin autofluorescence, which may be a better reflection of the extent of protein glycation $[16,17]$. The formation of AGE leads to their binding by a specific receptor class called receptor for advanced glycation endproducts (RAGE). This sets off proinflammatory cascades which have been linked to disease progression $[18,19]$. The AGE-RAGE role in disease states is not restricted to diabetes; numerous studies have linked advanced glycation endproducts to 
diseases ranging from osteoporosis to Alzheimer's disease [20-22]. In osteoporosis, it is thought that the accumulation of AGEs induces the formation of covalent cross-links with proteins (collagen) weakening the structural integrity of bone [23]. In addition, their binding to RAGE alters bone remodeling and leads to bone deterioration [24]. AGE can also be found in our diet, especially in meat products, and are absorbed in the gastrointestinal tract. Dietary AGE have been found to be an important contributor to the body AGE pool; this is especially observed when meat products are fried or barbecued [25].

While the phytochemicals in fruits and vegetables have similar bioactivities to that of The Food and Drug Administration (FDA) approved drugs that treat diabetes, polyphenolic compounds have one distinct property that is not observed with these glucose lowering agents. Polyphenolic compounds directly inhibit the non-enzymatic formation of advanced glycation endproducts; these molecules are capable of preventing the modification of the body's proteins independently of their effects of lowering plasma glucose concentrations [26,27]. Glycation is especially observed in long-lived proteins such as collagen, lens crystallins and hemoglobin [28].

Reducing sugars, such as glucose and fructose, form advanced glycation endproducts when incubated with protein; a process that can be followed by monitoring specific chemical markers. For example, the formation of Amadori products is an indication of the early phase of the process, while the formation of pentosidine is indicative of a late phase of AGE formation [29,30]. The major experimental approach to study the glycation process involves bovine serum albumin $(10 \mathrm{mg} / \mathrm{ml})$, a fairly soluble plasma protein, being incubated in the presence of $250 \mathrm{mM}$ glucose or fructose in the presence of phosphate buffer [31,32]. Since the physiological concentration of plasma glucose is less than $10 \mathrm{mM}$, the glycation process is greatly accelerated by the laws of mass action when the concentration of the reducing sugar is increased 25fold. After an incubation period of several days to two weeks at $37^{\circ} \mathrm{C}$, the glycation of the protein is detected by several different methods, ranging from the measurement of fluorescence intermediates at 370/440 $\mathrm{nm}$ to the measurement of a specific adduct of glycation, $\mathrm{N}$-(carboxymethyl) lysine [33]. There are several important characteristics of the non-enzymatic glycation process:
1. The glycation reaction is metal dependent; the metal-chelating agent diethylenetriaminepenta acetic acid (DETAPAC), reduced the covalent attachment of glucose to bovine serum albumin by approximately $50 \%[34,35]$.

2. The process can be significantly inhibited by the presence of antioxidants. The most studied group are polyphenolic compounds found in nature. Mastsuda and coworkers examined the relationship between the flavonoid structure, the inhibition of protein glycation and radical scavenging properties of sixty-two flavonoids [36]. Flavonoids with significant superoxide radical scavenging activing tended to be strong inhibitors of AGE formation. It has been recently found that ellagic acid, found in pomegranates and berries, can inhibit glycation by over $90 \%$ at concentrations less than $10 \mu \mathrm{M}[37,38]$.

3. Aldehydes, such as methylglyoxal, a well-studied intermediate in carbohydrate metabolism in mammals, are formed during the glycation of proteins. Methylglyoxal and other carbonyl groups, in the presence of a protein, can lead to the cross-linking associated with glycated proteins $[34,40]$ Many inhibitors of the glycation are capable of binding these carbonyl groups and prevent the cross-linking associated with glycated proteins. These agents "trap" carbonyls and prevent the formation of $\mathrm{N}$-(carboxymethyl) lysine [39]

4. Pharmacological agents have been developed that are called "cross-link breakers"; these agents break established AGE cross-links between proteins [41]. Alagebrium (3-phenacyl4,5-dimethylthiazolium chloride, ALT-711) decreases the complications associated with glycation; for example, alagebrium has been shown to decrease myocardial fibrosis and Improve ventricular function [42]. This bioactivity has also been documented for certain flavonoids, contributing to their ability to decrease the glycation burden [41]. While clinical trials have been performed on these "cross-line breakers", these studies have not resulted in any drug being approved by the FDA.

\section{CONCLUSION}

Evidence for the benefits of plant based foods to help control diabetes and the progression of diabetes 
related complications continues to grow. Clinicians should be aware of the dietary choices that may benefit patients with diabetes. Pharmacists are in a unique position to educate the public and the health care team on the application of appropriate use of medications and diet in patients with diabetes. Pharmacists are also able to take into account the pharmacodynamic parameters of dietary compounds and proteins. It is important for the pharmacist to advocate for a teambased approach when it comes to the appropriate management of diabetes, recognizing the role of both pharmacologic and non-pharmacologic modalities in the treatment of the disease state. Fruits and vegetables have long been known to be a beneficial component of a well-balanced diet. However, the intricacies of the mechanisms behind their benefits in patients with diabetes has not been previously described in detail.

Evidence suggests that the incorporation of these fruits and vegetables into ones' diet can lead to better outcomes and reduction of both microvascular and macrovascular complications in patients with diabetes. For patients, the explanation of these benefits and reduction of related comorbidities and overall mortality will likely be motivating in their efforts to make dietary adjustments and become more self-empowered in the management of their disease states.

\section{DECLARATIONS OF INTEREST}

None.

\section{FUNDING SOURCES}

No such involvement by authors.

\section{REFERENCES}

[1] Lifestyle Management: Standards of Medical Care in Diabetes- 2021. American Diabetes Association. Diabetes Care Jan 2019, 42 (Supplement 1) S1-S2; https://doi.org/10.2337/dc19-Sint01

[2] American Association of Clinical Endocrinologists and American College of Endocrinology Clinical Practice Guidelines for Developing a Diabetes Mellitus Comprehensive Care Plan-2015. Endocrine Practice 2015, 21 (Supplement 1). https://doi.org/10.4158/EP15672.GLSUPPL

[3] Mahoney, Sara \& Loprinzi, Paul. (2014). Influence of Flavonoid-Rich Fruit and Vegetable Intake on Diabetic Retinopathy and Diabetes-Related Biomarkers. Journal of Diabetes and its Complications. 28. https://doi.org/10.1016/j.jdiacomp.2014.06.011

[4] $\mathrm{Du} \mathrm{H}, \mathrm{Li} \mathrm{L}$, Bennett D, et al. Fresh fruit consumption in relation to incident diabetes and diabetic vascular complications: A 7-y prospective study of 0.5 million Chinese adults. PLoS Med. 2017;14(4):e1002279. Published 2017 Apr 11.

https://doi.org/10.1371/journal.pmed.1002279
[5] Chaudhury A, Duvoor C, Reddy Dendi VS, et al. Clinical Review of Antidiabetic Drugs: Implications for Type 2 Diabetes Mellitus Management. Front Endocrinol (Lausanne). 2017;8:6. Published 2017 Jan 24. https://doi.org/10.3389/fendo.2017.00006

[6] Ikeda, M., Shimazawa, R. Challenges to hemoglobin A1c as a therapeutic target for type 2 diabetes. J Gen Fam Med. 2019; 20(4): 129-138. https://doi.org/10.1002/jgf2.244

[7] Kumar S, Narwal S, Kumar V, Prakash O. a-glucosidase inhibitors from plants: A natural approach to treat diabetes. Pharmacogn Rev. 2011;5(9):19-29. https://doi.org/10.4103/0973-7847.79096

[8] McDougal, DJ., et al. Different polyphenolic component or soft fruits inhibit alpha-amylase and alpha-glucosidase. $J$ Agric Food Chem. 2005; 53(7): 2760-2766.

https://doi.org/10.1021/jf0489926

[9] Kalra S. Sodium Glucose Co-Transporter-2 (SGLT2) Inhibitors: A Review of Their Basic and Clinical Pharmacology. Diabetes Ther. 2014;5(2):355-66. https://doi.org/10.1007/s13300-014-0089-4

[10] Hardman, TC., Rutherford, P., Dubrey, SW., Wierzbicki, AS., Sodium-glucose co-transporter 2 inhibitors: from apple tree to 'sweet pee.' Curr Pharm Des. 2010: 16(34): 3830-3838. https://doi.org/10.2174/138161210794455111

[11] Tian C, Ye X, Zhang R, Long J, Ren W, et al. Green Tea Polyphenols Reduced Fat Deposits in High Fat-Fed Rats. PLOS ONE. 2015; 8(9): 10.

[12] Hurtado de llera A, Martin-hidalgo D, Rodriguez-gil JE, Gil MC, Garcia-marin LJ, Bragado MJ. AMP-activated kinase, AMPK, is involved in the maintenance of plasma membrane organization in boar spermatozoa. Biochim Biophys Acta. 2013;1828(9):2143-51.

https://doi.org/10.1016/j.bbamem.2013.05.026

[13] Sharma, C., et al. Advanced glycation end-products (AGEs): an emerging concern for processed food industries. J Food Sci Technol. 2015; 52(12): 7561-7576. https://doi.org/10.1007/s13197-015-1851-y

[14] Tessier FJ. The Maillard reaction in the human body. The main discoveries and factors that affect glycation. Pathol Biol (Paris) . 2010;58:214-9.

https://doi.org/10.1016/j.patbio.2009.09.014

[15] Ansari, Nadeem \& Moinuddin, Anis Shaikh \& Mir, Abdul Rouf \& Habib, Safia \& Alam, Khursheed \& Ali, Asif \& Khan, Rizwan Hasan. (2014). Role of Early Glycation Amadori Products of Lysine-Rich Proteins in the Production of Autoantibodies in Diabetes Type 2 Patients. Cell biochemistry and biophysics. 70 . https://doi.org/10.1007/s12013-014-9991-7

[16] Singh VP, Bali A, Singh N, Jaggi AS. Advanced glycation end products and diabetic complications. Korean J Physiol Pharmacol. 2014;18(1):1-14.

https://doi.org/10.4196/kjpp.2014.18.1.1

[17] Banser, A., Naafs, J., et al. Advanced glycation end products, measured in skin, vs. HbA1c in children with type 1 diabetes mellitus. Pediatr Diabetes. 2016; 17(6): 426-432. https://doi.org/10.1111/pedi.12311

[18] Stirban A, Gawlowski T, Roden M. Vascular effects of advanced glycation endproducts: Clinical effects and molecular mechanisms. Mol Metab. 2013;3(2):94-108. Published 2013 Dec 7. https://doi.org/10.1016/j.molmet.2013.11.006

[19] Fishman, SL., Sonmez, H., et al. The role of advanced glycation end-products in the development of coronary artery disease in patients with and without diabetes mellitus: a review. Mol Med 2018; 59(24).

https://doi.org/10.1186/s10020-018-0060-3 
[20] Kong, Y., Liu, C., Progress of RAGE molecular imaging in Alzheimer's Disease. Front Aging Neurosci. 2020; 12(227). https://doi.org/10.3389/fnagi.2020.00227

[21] Asadipooya, K., Mae EU. Advanced glycation end products (AGEs), receptor for AGEs, diabetes, and bone: review of the literature. Journal of the Endocrine Society. 2019; 3(10): 1799-1818.

https://doi.org/10.1210/js.2019-00160

[22] Luevano-Contreras, C., Chapman, K. Dietary advanced glycation endproducts and aging. Nutrients. 2010: 2(12): 1247-1265.

https://doi.org/10.3390/nu2121247

[23] Sanguineti, R., et al. Advanced glycation end products play adverse proinflammatory activities in osteoporosis. Mediators Inflamm. 2014; 2014: 975872. https://doi.org/10.1155/2014/975872

[24] Zhou, Z., Xiong, WC. RAGE and its ligands in bone metabolism. 2011; 3: 768-776. https://doi.org/10.2741/s185

[25] Uribarri, J., Woodruff, S., et al. Advanced glycation end products in foods and a practical guide to their reduction in the diet. J Am Diet Assoc. 2010; 110(6): 911-916. https://doi.org/10.1016/j.jada.2010.03.018

[26] Gkogkolou P, Böhm M. Advanced glycation end products: Key players in skin aging? Dermatoendocrinol. 2012;4(3):259-70.

https://doi.org/10.4161/derm.22028

[27] Chen, JH., Lin, X., et al. Role of advanced glycation end products in mobility and considerations in possible dietary and nutritional intervention strategies. Nutr Metab. 2018; 15:72. https://doi.org/10.1186/s12986-018-0306-7

[28] Gruyter, D. Advanced glycation end products mediated cellular and molecular events in the pathology of diabetic nephropathy. BioMol Concepts. 2016: 7(5): 293-309. https://doi.org/10.1515/bmc-2016-0021

[29] Perrone, A., et al. Advanced glycation end products (AGE): biochemistry, signaling, analytical methods, and epigenetic effects. Oxid Med Cell Longev. 2020; 2020. https://doi.org/10.1155/2020/3818196

[30] Ashraf, JM., et al. Recent advanced in detection of AGEs: immunochemical, bioanalytical and biochemical approaches. IUBMB Life. 2015: 67(12): 897-913. https://doi.org/10.1002/iub.1450

[31] Suantawee, T., et al. Protein glycation inhibitor activity and antioxidant capacity of clove extract. J Food Sci Tenchnol. 2015; 52(6): 3843-3850.
Rubio-Ruiz, ME., Glycation does not modify bovine serum albumin (BSA)-induced reduction of rat aortic relaxation: The response to glycated and nonglycated BSA is lost in metabolic syndrome. Glycobiology. 2008; 18(7): 517-525. https://doi.org/10.1093/glycob/cwn034

[33] Kinoshita, S., et al. $\mathrm{N}^{\omega}$-(Carboxymethyl)arginine is one of the dominant advanced glycation end products in glycated collagens and mouse tissues. Oxidative Medicine and Cellular Longevity. 2019. https://doi.org/10.1155/2019/9073451

[34] Salahuddin P, Rabbani G, Hasan Khan R. The role of advanced glycation end products in various -types of neurodgenerative disease: A therapeutic approach. Cell Mol Biol Lett.2014; 19: 407-437. https://doi.org/10.2478/s11658-014-0205-5

[35] Sadowska-Bartosz, I., et al. Kinetics of glycoxidation of bovine serum albumin by glucose, fructose and ribose and its prevention by food components. Molecules. 2014; 19(11): 18828-18849. https://doi.org/10.3390/molecules 191118828

[36] Matsuda, H.; Wang, T.; Managi, H.; Yoshikawa, M. Structura requirements of flavonoids for inhibition of protein glycation and radical scavenging activities. Bioorg. Med. Chem., 2003, $11,5317-5323$ https://doi.org/10.1016/j.bmc.2003.09.045

[37] Dorsey PG, Greenspan P. Inhibition of nonenzymatic protein glycation by pomegranate and other fruit juices. J Med Food. 2014;17(4):447-54. https://doi.org/10.1089/jmf.2013.0075

[38] Lim, TK. Punica Granatum. Edible Medicinal and NonMedicinal Plants. 2012;5: 136-194. https://doi.org/10.1007/978-94-007-4053-2 20

[39] Fournet, M., et al. Glycation damage: a possible hub for major pathophysiological disorders and aging. Aging Dis. 2018; 9(5): 880-900.

https://doi.org/10.14336/AD.2017.1121

[40] Mir AR, et al. Methylglyoxal mediated conformational changes in histone $\mathrm{H} 2 \mathrm{~A}$-generation of carboxyethylated advanced glycation end products. Int J Biol Macromol, 2014 69:260-6.

https://doi.org/10.1016/j.ijbiomac.2014.05.057

[41] Snedeker, JG., Gautieri, A., The role of collagen crosslinks in aging and diabetes - the good, the bad, and the ugly. Muscles Ligaments Tensons J. 2014: 4(3): 303-308. https://doi.org/10.11138/mltj/2014.4.3.303

[42] Toprak, C., Yigitaslan, S., Alagebrium and complications of diabetes mellitus. Eurasian J Med. 2019; 51(3): 285-292. https://doi.org/10.5152/eurasianjmed.2019.18434 